\title{
Efectos de una intervención siguiendo el modelo enfocado en la familia para promover avances en el desarrollo del lenguaje de los niños
}

\author{
Camila Domeniconi ${ }^{1}$ y Marta Gràcia ${ }^{2}$
}

Recibido: 23 de enero de 2018 / Revisado: 12 de marzo de 2018 / Aceptado: 14 de abril de 2018

\begin{abstract}
Resumen. La intervención siguiendo el modelo enfocado en la familia, como camino hacia las prácticas centradas en la familia, aprovecha las situaciones naturales de las rutinas diarias para promover el desarrollo de los niños. El estudio ha tenido como objetivo implementar y evaluar la eficacia de una intervención centrada en cuatro familias para conseguir el avance en las habilidades comunicativas de sus hijos de cinco años, medidas a través de pruebas estandarizadas de lenguaje, y de análisis de actividades comunicativas con los padres y con la investigadora. Las familias participaron en cinco reuniones donde se discutieron estrategias útiles para ayudar a los niños a superar nuevos retos comunicativos. El análisis de los datos pone de relieve que todos los niños han mejorado sus habilidades comunicativas, en todas las valoraciones. Asimismo, reflejan una vez más la importancia de la interacción comunicativa entre adultos y niños, y el potencial de una intervención siguiendo un modelo enfocado en la familia para promover avances en el desarrollo comunicativo de los niños.
\end{abstract}

Palabras clave: intervención siguiendo el modelo enfocado en la familia; lengua oral; educación infantil; empoderamiento

\section{[En] Effects of a family-focused intervention model to promote the development of children's oral language}

\begin{abstract}
The family-focused model interventions, as a way towards the family-centered intervention practices, takes advantage of the natural situations of daily routines to promote the development of children. The objective of the study was to implement and evaluate the effectiveness of an intervention focused on four families to achieve progress in the communication skills of their five-year-old children, measured through standardized language tests, and analysis of communicative activities with the children, parents and with the researcher. Families participated in five meetings where useful strategies to help children to achieve new communication challenges were discussed. The analysis of the data highlights that all children have improved their communication skills, in all assessments. They also reflect once again the importance of communicative interaction between adults and children, and the potential of a family-focused intervention model to promote advances in the communicative development of children.
\end{abstract}

Key words: family-focused intervention model; oral language; early childhood education; empowerment

Cómo citar: Domeniconi, C., y Gràcia, M. (2018). Efectos de una intervención siguiendo el modelo enfocado en la familia para promover avances en el desarrollo del lenguaje de los niños. Revista de Investigación en Logopedia, 8(2), 165-181.

1 Centro de Educação e Ciências Humanas, Departamento de Psicologia. Universidade Federal de São Carlos. Brasil.

domeniconicamila@gmail.com

2 Departamento de Cognición, Desarrollo y Psicología de la Educación. Universidad de Barcelona. España. 


\section{Introducción}

Las dificultades de los niños en la adquisición del lenguaje oral han sido consideradas como un factor de riesgo para futuras dificultades sociales y académicas. Investigaciones llevadas a cabo desde distintos marcos teóricos tienden a valorar la interacción entre adultos y niños positivamente para el impulso del lenguaje oral y de las interacciones comunicativas (Hoff, 2012; Tomasello, 2003).

Los padres y madres han tenido un papel fundamental en los programas de intervención con el objetivo de promover la lengua oral, el vocabulario, la comprensión y la prevención de la aparición de problemas de aprendizaje (Benitez \& Domeniconi, 2012, 2014; Gràcia, 2001; Gortmaker, McCurdy, Persampieri, \& Hergenrader, 2007; Gurgueira \& Cortegoso, 2008; Hancock \& Kaiser, 2002; Hart \& Risley, 1995; Vilaseca \& del Rio, 2004). Sin embargo, una variable crítica que ha sido identificada como una dificultad en estos trabajos es que la familia, muchas veces, no parece involucrarse suficientemente para contribuir a mejorar las dificultades de los niños o para promover avances importantes (Stocco \& Thompson, 2015).

Uno de los motivos que explican la insuficiente implicación de la familia en las intervenciones tiene relación con el hecho de que el planteamiento de las intervenciones no suele tener en cuenta la rutina familiar para facilitar que los procedimientos propuestos sean incorporados por las familias. Adaptar los procedimientos a la rutina familiar, tener en cuenta el conocimiento que las familias tienen sobre su hijo y sobre su contexto, es necesario para potenciar los efectos de las intervenciones y, además, para ayudar a la familia a progresar, a empoderarse, incluso más allá de la finalización de la intervención centrada en la familia.

Los procedimientos naturales tienen la ventaja de considerar este requisito, puesto que se aprovechan las situaciones naturales de la rutina diaria para intervenir $y$, así, se garantiza la funcionalidad de la situación y el mantenimiento de las relaciones comunicativas y de las conductas que suscitan interés por parte de los niños (Dempsey \& Keen, 2008; Espe-Sherwindt \& Serrano, 2016; García-Sánchez, Escorcia Mora, Sánchez López, Orcajada Sánchez, \& Hernández-Pérez, 2014; Hart, \& Rogers-Waren, 1978; Koegel, O’Dell, \& Koegel, 1987).

En general, se considera que la mayor parte de las familias tienen como rutina la lectura de cuentos con sus hijos pequeños. Este hábito puede llegar a ser muy importante para contribuir a la enseñanza y aprendizaje de contenidos relacionados con la lengua oral y escrita. Además, mirar cuentos conjuntamente es una oportunidad para mantener una conversación tranquila entre adultos y niños, y así promover avances en el vocabulario y las habilidades lingüísticas de los niños, incluso los que viven en condiciones socioeconómicas menos favorables o más vulnerables (Dickinson \& Tabors, 2001; Milburn, Girolametto, Weitzman, \& Greenberg, 2014).

Colmar (2014) investigó el impacto de un programa de intervención con los padres y madres teniendo como base la lectura de cuentos a los niños que vivían en condiciones socioeconómicas desfavorables. En el programa participaron 36 niños distribuidos en un grupo control (24) y un grupo de intervención (11). Todos los niños del grupo de intervención y la mitad de los niños del grupo control (12 niños) presentaban niveles de lenguaje por debajo del esperado para su edad. En el grupo control, la otra mitad de los niños presentaban un desarrollo normal del lenguaje. En el programa de intervención los padres y madres aprendieron a interactuar con sus hijos mientras miraban cuentos juntos, utilizando cuatro pautas básicas: 1. Hacer 
pausas en cada página del cuento (el objetivo era que el niño tuviera la oportunidad de hablar sobre lo que le interesaba, mientras miraba los dibujos o imágenes en el cuento); 2. Formular preguntas abiertas a los niños sobre lo que decían mientras miraban los cuentos; 3. Utilizar los dibujos como base para incentivar que los niños hablasen, si los niños no lo hacían espontáneamente; 4. Intentar hablar sobre el tema de los cuentos, las palabras nuevas que aparecían, o utilizando nuevas estructuras de frases, a lo largo del día después de la lectura. Después de cuatro meses de intervención todos los niños fueron valorados con las mismas pruebas que al inicio. Los resultados ponen en evidencia que los niños del grupo intervención mejoraron de manera significativa respecto a los niños del grupo control.

La claridad del procedimiento y la importancia de los resultados obtenidos por Colmar (2014) ponen de manifiesto que una intervención como la que se implementa podría beneficiar a niños con distintas condiciones socioeconómicas y también con distintos perfiles de desarrollo comunicativo. En base a los resultados de Colmar (2014) nos podemos preguntar cómo son las interacciones comunicativas que ocurren naturalmente entre los padres y los niños en sus actividades durante las rutinas diarias, como mirar cuentos, y en qué sentido pueden influir en la eficacia de un programa de intervención como el que se presenta.

Con el objetivo de disponer de una herramienta para valorar las estrategias y los patrones de comunicación que se dan en las familias, hemos desarrollado y utilizado en la presente investigación una adaptación de la Escala de Valoración de la Lengua Oral en contexto Escolar - EVALOE - (Gràcia et al., 2015; Gràcia, Vega, Galván-Bovaira, 2015), para el contexto familiar (Escala EVALOF - Escala de Valoración de la Lengua Oral en contexto Familiar) (Domeniconi, Gràcia, Benitez, \& Vessoni, 2017). La escala EVALOE ha sido utilizada por los docentes para promover el desarrollo de la competencia oral en el aula, y su adaptación para el contexto familiar tiene los mismos objetivos, esto es, valorar cómo son las interacciones comunicativas entre los padres y sus hijos en casa. En este caso también se utiliza la herramienta como instrumento de intervención, usando los ítems de la escala como base en procesos de asesoramiento. La escala es una herramienta de observación que explora la interacción entre padres y sus hijos mientras realizan actividades diversas en sus hogares, y está formada por dos partes. La primera parte comprende 11 ítems que fueron diseñados para evaluar el Contexto y gestión de la comunicación; y la segunda, que tiene 21 ítems, fue construida para valorar Las funciones y estrategias comunicativas.

Se construyó con el objetivo de crear una herramienta flexible y capaz de identificar y valorar los aspectos clave de la interacción entre los niños y los adultos de su familia. Pretendemos que la identificación de estos aspectos clave de interacción resulte útil para comprender posibles variables importantes en el éxito de los procedimientos de intervención planteados, por ejemplo, en la investigación de Colmar (2014) a la que nos hemos referido, e incluso para la detección de posibles cambios que puedan ocurrir al largo del proceso de intervención con la familia.

Considerando la importancia de la promoción del lenguaje oral como vehículo de comunicación y como factor de protección contra fracasos futuros o exclusión social y académica, y considerando la necesidad de herramientas que ayuden a evaluar los patrones de interacción comunicativa que tienen lugar en el contexto familiar, el presente estudio ha tenido como objetivo implementar y evaluar la eficacia de una intervención centrada en las familias para el avance en las habilidades comunicativas de los niños, medidas a través de pruebas estandarizadas de lenguaje, y análisis de la 
conducta en actividades comunicativas con pares y con la investigadora. Teniendo en cuenta la potencialidad de la actividad de mirar cuentos para el avance en el desarrollo de la lengua oral, la intervención fue planificada para que el foco se situase en la actividad de mirar cuentos, aunque las pautas del procedimiento de intervención fuesen útiles para muchos otros momentos de interacción comunicativa entre los niños y su familia. Como un segundo objetivo, nos proponemos valorar la utilidad de la EVALOF (Escala de Valoración de la enseñanza de la Lengua Oral en contexto Familiar (Domeniconi, Gràcia, Benitez, \& Vessoni, 2017) como una herramienta para valorar cómo interactúan los padres antes y después de la intervención, con el fin de construir un conocimiento profundo de las condiciones que son necesarias y suficientes para el éxito de una intervención como la que planteamos en nuestro estudio.

\section{Metodología}

\section{Participantes}

Han participado cuatro familias con al menos un hijo de cinco años con desarrollo típico que viven en el extrarradio de una ciudad de Cataluña de 200.000 habitantes. Dos de los niños son hermanos gemelos. A través de la dirección de la escuela las familias fueron invitadas a participar en la investigación. Concretamente, se inició un primer contacto con la escuela y se les explicó nuestro interés en realizar una investigación con el fin de ayudar a familias a mejorar sus pautas de interacción con sus hijos del último curso de educación infantil. No se plantearon criterios de exclusión. La dirección de la escuela se reunió con las familias que consideraba que estarían interesadas en participar y el resultado fue la selección de cuatro familias. La Tabla 1 contiene una caracterización de cada una de las familias participantes.

Tabla 1. Caracterización de las familias. Se incluyen informaciones sobre la edad, profesión, nivel de escolaridad de los miembros de la familia que viven con los niños, $\mathrm{y}$ lenguas de uso en casa.

\begin{tabular}{lllll}
\hline & $\begin{array}{l}\text { Juan y Olavo } \\
\text { (gemelos) }\end{array}$ & Daniela & Sandra & Ana \\
\hline $\begin{array}{l}\text { Personas que convi- } \\
\text { ven en el domicilio } \\
\text { familiar (además del } \\
\text { niño) }\end{array}$ & & $\begin{array}{l}\text { Madre, padre y herma- } \\
\text { na mayor }\end{array}$ & Madre y padre & Madre y padre \\
\hline $\begin{array}{l}\text { Edad de madre / } \\
\text { padre }\end{array}$ & $44 / 44$ & $37 / 44$ & $38 / 35$ & $35 / 33$ \\
\hline $\begin{array}{l}\text { Profesión madre/ } \\
\text { padre }\end{array}$ & $\begin{array}{l}\text { Peluquera / informá- } \\
\text { tico }\end{array}$ & $\begin{array}{l}\text { Ama de la casa / me- } \\
\text { cánico }\end{array}$ & $\begin{array}{l}\text { Dependienta/ } \\
\text { troquelador }\end{array}$ & Fábrica/ carpintero \\
\hline $\begin{array}{l}\text { Escolaridad } \\
\text { Grado (FP)/ }\end{array}$ & $\begin{array}{l}\text { Bachillerato/ } \\
\text { Grado medio }\end{array}$ & $\begin{array}{l}\text { Grado medio / } \\
\text { nado) }\end{array}$ & $\begin{array}{l}\text { Enseñanza básica / } \\
\text { Grado medio }\end{array}$ \\
\hline $\begin{array}{l}\text { Lenguas de uso en } \\
\text { casa }\end{array}$ & Castellano & Árabe & Castellano & Castellano \\
\hline
\end{tabular}




\section{Procedimiento}

\section{Diseño}

Se trata de un estudio de casos con medidas tomadas durante la intervención y también medidas antes y después de la intervención (Yin, 2008). Como unidades de análisis hemos elegido los momentos de interacción comunicativa de los niños con sus familias, especialmente los momentos de mirar cuentos. En el análisis de datos nos hemos centrado en las habilidades lingüísticas de los niños y en las interacciones comunicativas entre los padres y sus hijos durante sus rutinas diarias.

\section{Instrumentos}

a) Para poder analizar los cambios en las habilidades comunicativas y lingüísticas de los niños se utilizaron los siguientes instrumentos:

1. Prueba de Lenguaje Oral Navarra (PLON) (Blázquez, Ayerra, Suso, Bidegain, Baquedano, 2004) y el módulo de Pragmática del BLOC Screening Revisado (BLOC-S-R) (Puyuelo, Renom, Solanas \&, Wiig, 2007), para evaluar el lenguaje de los niños. Los dos instrumentos han sido aplicados de acuerdo con las normas y los baremos de las pruebas, las aplicaciones han sido grabadas en audio y analizadas posteriormente.

2. Instrumento para valorar las habilidades de conversación de los niños. El instrumento para valorar las habilidades de conversación de los niños en su ambiente natural ha sido desarrollado en el marco del proyecto Los sistemas de soporte a la toma de decisiones docentes (SSD) y el desarrollo de la competencia lingüistica (EDU2015-63616-P) (Gràcia, 2017). El uso del instrumento permite valorar habilidades no verbales (4 ítems) y verbales (18 ítems agrupados en 6 ámbitos de habilidad comunicativa: gestión de turnos, coherencia, funciones comunicativas, discurso argumentativo, aspectos formales y uso de la lengua vehicular de la escuela) de cada uno de los niños involucrados en la conversación. Se trata de una conversación entre 3-5 niños que tiene lugar en la escuela y que tiene como consigna "discutid para llegar a un acuerdo sobre los detalles de la organización de una fiesta de cumpleaños para uno de los niños de vuestro grupo clase -al inicio de curso- y de una excursión de final de curso, -en la evaluación llevada a cabo al final de curso-".

3. Actividad de mirar cuentos. Los niños participaron en una actividad de mirar cuentos con la investigadora, antes y después de la intervención, que fue registrada en vídeo. La situación seguía siempre la misma estructura: 1) se invitaba al niño a mirar un cuento con la investigadora en una sala de la escuela; 2) la investigadora le presentaba tres opciones de cuentos (los tres muy parecidos en términos de tema, número de páginas y tipo de texto en cada página) de los que tenía que elegir uno; 3 ) a continuación, la investigadora leía en voz alta el título del libro, pasaba la primera página y esperaba a que el niño empezara a decir algo y a pasar las páginas; 4) al final de la actividad, la investigadora siempre pedía a los niños que intentasen hacer una síntesis del cuento. Las categorías medidas a partir de las grabaciones en vídeo de esta actividad fueron: 1. Manipulación: tiene como objetivo detectar si los niños manipulan los libros mientras están a su alcance; 2 . Aprovechar las pausas para hablar: mide si los niños aprovechan las pausas de la investigadora, en el momento en el que 
pasa la página del cuento; 3. Conectivos: en esta categoría se agrupan las habilidades que los niños han mostrado para contar una historia con una determinada secuencia en cada página, incluso con uso de conectivos (por ejemplo: entonces, luego...); 4. Finalizar: pensada para detectar si los niños finalizan la historia, con alguna lógica (con relación a la historia) o con la palabra fin; 5. Sintetizar: hace referencia a las intervenciones de los niños que indican su habilidad para incorporar la estrategia de síntesis; 6. Narración: la manera como los niños cuentan el cuento ha sido valorada de acuerdo con los parámetros de nombrar, describir o narrar; agrupa todos los datos sobre la habilidad narrativa, que es considerada la más esperada para la edad.

b) Para valorar las rutinas y las interacciones comunicativas de los padres y madres con sus hijos, se utilizaron los siguientes instrumentos:

1. Guion para llevar a cabo una entrevista con las familias sobre sus rutinas diarias basada en la propuesta por McWilliam (2016). Se aplicó antes de la intervención.

2. EVALOF (Domeniconi, Gràcia, Benitez, \& Vessoni, 2017). La EVALOF está formada por 32 ítems agrupados en dos subescalas: 1) Contexto y gestión de la comunicación (12 ítems); y 2) Funciones comunicativas y estrategias (20 ítems). Cada uno de los ítems puede ser valorado por el observador con una puntuación de 0 a 4 , siendo 0 - No se puede observar (esta opción debe ser elegida si por alguna razón, la acción no puede ser observada, por ejemplo, si la pregunta hace referencia a una interacción en red entre un pequeño grupo de personas, y solo estaban presentes dos personas, la acción no puede ser observada); 1 - no se observa la acción; 2 - se observa algunas veces; 3 - se observa sistemáticamente o casi siempre. La escala EVALOF ha sido construida a partir de la escala EVALOE (Escala para Valoración de la Enseñanza de la Lengua Oral en contexto Escolar (Gràcia et al., 2015). Esta versión de la EVALOF se completa a partir del visionado de un registro en vídeo de un episodio de una rutina natural de la familia. Para obtenerlo se pidió a las familias que registrasen en vídeo una actividad de su rutina que fuese lo más natural posible. Los registros fueron llevados a cabo antes y después de la intervención.

\section{Intervención centrada en la familia}

Las familias participaron en ocho reuniones con una de las investigadoras, siendo una primera reunión de contacto -en la que estaban presentes las dos investigadoras-, una entrevista sobre las rutinas de la familia, cinco reuniones de seguimiento con cada familia, y una reunión final de valoración de la intervención con las cuatro familias juntas. Las reuniones tuvieron lugar siempre con la investigadora y la madre (en el caso de Ana) o la madre y padre (en el caso de las otras tres familias). Cada familia decidió si prefería que la reunión tuviera lugar en casa o en la escuela. Las reuniones se celebraron una vez al mes, con una duración aproximada de 90 minutos. Las familias discutieron en la primera reunión un documento con la descripción de siete estrategias sencillas que se sugería a los padres y madres para las que intentasen poner en práctica en los momentos de mirar cuentos con los niños. Además, recibieron una propuesta de calendario con el objetivo de anotar los días en los que había sido posible para ellos mirar cuentos, así como para anotar alguna duda o dificultad que tuviesen durante la actividad de mirar cuentos con sus hijos. La intervención se inspiró en las pautas propuestas por Colmar (2014) respecto a la actividad de mirar cuentos, con algunas modificaciones introducidas a partir de lo que la familia había 
aportado en la entrevista sobre las rutinas de la familia respecto al tipo de actividades que llevaban a cabo con sus hijos en casa, puesto que en todos los casos, excepto uno, relataron que miraban cuentos con sus hijos en casa. Concretamente, las pautas fueron: 1. Hacer una pausa en cada página del cuento para dejar hablar al niño; 2. Seguir el interés del niño y concretamente la historia que está contando; 3 . Hacer preguntas abiertas; 4. Explicar de manera muy sencilla el significado de alguna nueva palabra que sea importante para la comprensión de la historia; 5. Promover el cambio de turno en las conversaciones; 6 . Elogiar los intentos comunicativos del niño y aprovechar lo que dice para continuar o expandir su producción; 7. Cuando sea posible, y de manera natural, recordar elementos de la historia, especialmente palabras nuevas, en otros momento del día. Cada una de las estrategias fue discutida con la familia, a partir de lo que tres de las cuatro las familias habían explicado en la entrevista de rutinas sobre cómo miraban cuentos con sus hijos y qué les parecía que más les gustaba y que mejor funcionaba para mantener la actividad. En el caso de la familia de los dos niños gemelos, no fue posible partir de la manera como solían mirar cuentos porque esta actividad no formaba parte de sus rutinas diarias. En este caso se intentó partir de otras actividades similares. Se pidió a las familias que intentasen practicar alguna de las estrategias, o más de una, en las semanas siguientes. Se enfatizó la importancia de animar al niño a hablar y seguir su interés, sin necesidad de que el texto del cuento o libro fuese seguido para que se contase o construyese una historia. También se sugirió el uso de las estrategias en cualquier momento de rutina de la familia, en la que fuese natural formular preguntas a los niños y mantener una conversación con ellos. Para promover avances en la lengua oral de los niños se compartieron con las familias las maneras de clarificar y de expandir las intervenciones realizadas por los niños. A continuación, las cuatro reuniones de seguimiento siguientes tuvieron como base las estrategias, la manera como cada una de las familias podían practicarlas, las dificultades que encontraban y sus sugerencias. Dado el carácter personalizado del asesoramiento, las reuniones no tuvieron exactamente los mismos retos para todas las familias, puesto que la investigadora se ajustaba a las características de cada una de ellas, aunque el objetivo siempre fuese el mismo, empoderarlas para ayudar a los niños a mejorar su competencia comunicativa oral. Los objetivos personalizados de cada una de las reuniones se pueden revisar en el Apéndice 1.

Después de las cinco reuniones con cada una de las familias, todas ellas participaron en una reunión de valoración en grupo. El objetivo de la última reunión fue reflexionar sobre todas las estrategias utilizadas, la experiencia y el trabajo llevado a cabo, valorar la percepción de las madres y padres, recoger ideas sobre elementos que introducirían en un proceso como el que habían participado si se tuviese que volver a empezar con otras familias, recoger la percepción de las familias sobre lo que consideraban que había cambiado en sus rutinas o en la manera como hablaban con sus hijos, las dificultades habían tenido, y cómo creían que las habían resuelto y las podrían continuar resolviendo. También se discutieron detalles sobre los procedimientos para la recogida de datos de la evaluación final, como las evaluaciones del lenguaje de los niños y las grabaciones en la escuela y en casa, y se proporcionaron sugerencias para continuar ayudando a los niños a implicarse en las actividades escolares en general, y especialmente aquellas relacionadas con la competencia lingüística. 


\section{Procedimiento de análisis de datos}

La Prueba de Lenguaje Oral Navarra (PLON) y el módulo de pragmática de la Batería de Lenguaje Objetiva y Criterial (BLOC) han sido analizadas de acuerdo con los criterios recogidos en el manual de aplicación de las pruebas. Todas las aplicaciones fueron registradas en audio, lo que permitió una revisión en caso de duda. Los registros en vídeo de la interacción comunicativa en el domicilio familiar fueron valorados por las investigadoras, y los ítems que presentaron dudas fueron discutidos, así como los resultados generales obtenidos con la aplicación de la EVALOF.

Las interacciones comunicativas entre los niños fueron valoradas de acuerdo con el instrumento ad hoc al cual ya nos hemos referido en el apartado de instrumentos: Instrumento para valorar las habilidades de conversación de los niños.

La actividad de mirar cuentos con una de las investigadoras fue registrada en vídeo y valorada de acuerdo con las siguientes categorías: 1. la manipulación del libro; 2. las producciones infantiles mientras la investigadora hace pausas; 3 . el uso de conectivos; 4. la finalización de la historia; 5 . habilidad de hacer un resumen o síntesis; 6. uso de narración.

Las entrevistas fueron registradas en audio y las dos investigadoras discutieron y seleccionaron conjuntamente los elementos principales que se recogen en el resumen de los principales aspectos relacionados con la interacción comunicativa en cada familia que se presenta en el siguiente apartado.

\section{Resultados}

1. Valoración de las rutinas y de la enseñanza de la lengua oral en contexto familiar

El primer resultado que se presenta es la descripción de las actividades que forman parte de la rutina de los niños. En la Tabla 2 se presentan los datos obtenidos en la entrevista basada en rutinas con cada familia.

Tabla 2. Descripción de las rutinas de las familias, de acuerdo con el relato de los padres al inicio de la recogida de datos, concretamente en la entrevista basada en rutinas (McWilliam, 2016).

\begin{tabular}{llllll}
\hline & Ana & Daniela & Olavo & Juan & Sandra \\
\hline $\begin{array}{l}\text { Actividades } \\
\text { durante la } \\
\text { mañana }\end{array}$ & $\begin{array}{l}\text { Algunas veces } \\
\text { desayunan } \\
\text { juntos, pero no } \\
\text { siempre }\end{array}$ & $\begin{array}{l}\text { Desayunan jun- } \\
\text { tos, se peinan, } \\
\text { se visten }\end{array}$ & $\begin{array}{l}\text { Leche en } \\
\text { biberón, } \\
\text { mirando la } \\
\text { tele }\end{array}$ & $\begin{array}{l}\text { Leche en bib- } \\
\text { erón, mirando } \\
\text { la tele }\end{array}$ & $\begin{array}{l}\text { Bebe la leche miran- } \\
\text { do la tele, se peina }\end{array}$ \\
\hline $\begin{array}{l}\text { Actividades du- } \\
\text { rante la tarde }\end{array}$ & $\begin{array}{l}\text { Casa de la abue- } \\
\text { la con prima } \\
\text { algunas veces, } \\
\text { inglés 1 vez a la } \\
\text { semana }\end{array}$ & $\begin{array}{l}\text { Colegio Árabe } \\
\text { 2 veces a la } \\
\text { semana, otros } \\
\text { días parque y } \\
\text { deberes en casa }\end{array}$ & $\begin{array}{l}\text { Juegan, } \\
\text { meriendan, } \\
\text { ducha }\end{array}$ & $\begin{array}{l}\text { Juegan, me- } \\
\text { riendan, ducha }\end{array}$ & $\begin{array}{l}\text { Casa de la abuela al- } \\
\text { gunas veces (primos } \\
\text { y primas), inglés 1 }\end{array}$ \\
& & & & una vez a la semana \\
\end{tabular}




\begin{tabular}{|c|c|c|c|c|c|}
\hline $\begin{array}{l}\text { Actividades du- } \\
\text { rante festivos o } \\
\text { fines de semana }\end{array}$ & $\begin{array}{l}\text { Salen mucho, } \\
\text { visitan a la fa- } \\
\text { milia y amigos, } \\
\text { cine, biblioteca }\end{array}$ & $\begin{array}{l}\text { Van de com- } \\
\text { pras, algunos } \\
\text { días parque, } \\
\text { hacen deberes } \\
\text { los domingos }\end{array}$ & $\begin{array}{l}\text { No salen } \\
\text { mucho, } \\
\text { visitan el piso } \\
\text { del abuelo y } \\
\text { comen con } \\
\text { el tío }\end{array}$ & $\begin{array}{l}\text { No salen } \\
\text { mucho, visitan } \\
\text { el piso del } \\
\text { abuelo y } \\
\text { comen con } \\
\text { el tío }\end{array}$ & $\begin{array}{l}\text { Salen mucho, } \\
\text { viajan cuando tienen } \\
\text { dinero, visitan a la } \\
\text { familia }\end{array}$ \\
\hline $\begin{array}{l}\text { Momentos } \\
\text { en los que } \\
\text { acostumbran a } \\
\text { hablar }\end{array}$ & $\begin{array}{l}\text { En el coche, } \\
\text { cuando llegan a } \\
\text { casa, en muchos } \\
\text { momentos }\end{array}$ & $\begin{array}{l}\text { Durante las co- } \\
\text { midas y cuando } \\
\text { hacen deberes }\end{array}$ & $\begin{array}{l}\text { Cuando } \\
\text { caminan } \\
\text { volviendo de } \\
\text { la escuela (en } \\
\text { general van } \\
\text { en coche) }\end{array}$ & $\begin{array}{l}\text { Cuando cami- } \\
\text { nan volviendo } \\
\text { de la escuela } \\
\text { (en general } \\
\text { van en coche) }\end{array}$ & $\begin{array}{l}\text { Caminando hacia } \\
\text { zumba, en algunas } \\
\text { comidas, la niña } \\
\text { habla todo tiempo }\end{array}$ \\
\hline $\begin{array}{l}\text { Actividades } \\
\text { preferidas de } \\
\text { los niños }\end{array}$ & $\begin{array}{l}\text { Bailar, dibujar, } \\
\text { mirar cuentos }\end{array}$ & $\begin{array}{l}\text { Parque, salir en } \\
\text { coche }\end{array}$ & $\begin{array}{l}\text { Actividades } \\
\text { movidas } \\
\text { (pelota, } \\
\text { patines...) }\end{array}$ & $\begin{array}{l}\text { Actividades } \\
\text { movidas (pelo- } \\
\text { ta, patines...) }\end{array}$ & $\begin{array}{l}\text { Manualidades, } \\
\text { dibujos }\end{array}$ \\
\hline $\begin{array}{l}\text { ¿Miran cuentos } \\
\text { juntos? }\end{array}$ & $\begin{array}{l}\text { Sí, por las } \\
\text { noches }\end{array}$ & $\begin{array}{l}\text { Sí, mientras } \\
\text { hacen deberes }\end{array}$ & No & No & $\begin{array}{l}\text { Sí, antes de ir a } \\
\text { dormir }\end{array}$ \\
\hline
\end{tabular}

En los resultados presentados en la Tabla 2 se pone de relieve el grado en que las familias aprovechan las actividades de sus rutinas diarias para hablar con los niños. Por ejemplo, en las comidas, únicamente la familia de Daniela aprovecha para estar juntos y hablar. Las actividades más elegidas por las familias para hablar son las que implican un traslado, por ejemplo, de casa a la escuela o viceversa. Por otro lado, tres de las cuatro familias acostumbran a mirar cuentos con los niños, y esta actividad forma parte de su rutina, lo que es un indicativo de que una intervención centrada en esta actividad puede que sea adecuada para respetar la idea de plantear la intervención a partir de las rutinas de las familias, partiendo de las estrategias que ya utilizan, y evitar plantear actividades o tareas complejas y poco naturales, alejadas de las rutinas de las familias.

La Tabla 3 pone de manifiesto que las familias se han implicado en promover algunos cambios en la manera cómo interactúan y enseñan la lengua oral a sus hijos. En todas las familias se observan porcentajes más elevados en las medidas tomadas después de la intervención, respecto a las medidas tomadas antes de la intervención.

Tabla 3. Porcentaje total obtenido en la EVALOF a partir de las observaciones de los episodios comunicativos de la familia, antes y al final de la intervención.

\begin{tabular}{lcc}
\hline & Inicial & Final \\
\hline Ana & EVALOF \% & EVALOF \% \\
Daniela & 46 & 64 \\
Juan y Olavo & 57 & 62 \\
Sandra & 52 & 66 \\
\hline \multicolumn{3}{c}{}
\end{tabular}


Los ítems de la EVALOF que han cambiado son distintos para cada familia. Por ejemplo, en la familia de los dos hermanos gemelos, se han incrementado mucho más las estrategias de clarificación y expansión, y han dado mucho más tiempo y espacio a los niños para participar en la conversación, manteniendo conversaciones más abiertas con ellos, más dirigidas por los niños, menos dirigidas por los adultos. Por otro lado, las familias de las tres niñas tenían, en la primera evaluación, una tendencia a dejar que las niñas hablasen sin casi ningún tipo de interacción con ellas, simulando que eran maestras en la escuela, o como si las niñas estuviesen solas. En el análisis final, se han observado más intentos de pedir autoevaluaciones y síntesis, de hacer preguntas abiertas, lo que permite que los padres enseñen otros contenidos lingüísticos, mientras las niñas dirigen la conversación.

2. Valoración de las habilidades comunicativas de los niños antes y después de la intervención

Tabla 4. Resultados de la evaluación inicial y final en las diversas pruebas aplicadas a cada uno de los niños.

\begin{tabular}{lccccc}
\hline & Juan & Olavo & Ana & Daniela & Sandra \\
\hline PLON* (13 ítems) & & & & & \\
$\quad$ Inicial & $\mathrm{R}$ & $\mathrm{R}$ & $\mathrm{N}$ & $\mathrm{NM}$ & $\mathrm{NM}$ \\
$\quad$ Final & $\mathrm{N}$ & $\mathrm{N}$ & $\mathrm{N}$ & $\mathrm{N}$ & $\mathrm{N}$ \\
\hline $\begin{array}{l}\text { BLOC (23 ítems) } \\
\text { Inicial }\end{array}$ & 5 & 8 & 9 & 4 & 15 \\
$\quad$ Final & $10^{* *}$ & 14 & 9 & 9 & 21 \\
$\begin{array}{l}\text { Conversación } \\
\text { (57 indicadores) }\end{array}$ & & & & & \\
$\quad$ Inicial & 6 & 3 & 6 & 4 & 5 \\
$\quad$ Final & 42 & 37 & - & 33 & 43 \\
\hline
\end{tabular}

* R: Retraso; NM: Necesita mejorar; N: Normal

** la investigadora no termina la aplicación de la prueba porque el niño no parecía cómodo.

En la Tabla 4 se puede observar que las puntuaciones de las pruebas se incrementan en la evaluación final respecto a la inicial. Concretamente, los resultados de las dos aplicaciones de la Prueba de Lenguaje Oral Navarra (PLON) (Blázquez et. al, 2004) ponen de relieve que cuatro de los cinco niños presentaban en la primera evaluación medidas de lenguaje por debajo de lo esperado para su edad cronológica. Juan y Olavo obtienen puntuaciones compatibles con lo que los autores de la prueba consideran un Retraso en Lenguaje (RL), y Sandra y Daniela presentan niveles compatibles con Necesidad de Mejorar (NM). Después de la intervención con las familias, todos los niños han alcanzado resultados compatibles con lo esperado para su edad, de acuerdo con los parámetros de la prueba PLON. Respecto a la valoración del módulo de pragmática de la Batería de Lenguaje Objetiva y Criterial (BLOC) (Puyuelo, et.al, 2007), también se ha observado que casi todos los niños han mejorado notablemente sus puntuaciones en la evaluación final, respecto a la inicial, con excepción de Ana, que ha mantenido la misma puntuación en las dos valoraciones. 
Juan, por ejemplo, aunque no haya terminado la prueba, ha duplicado la puntuación en la última valoración respecto a la inicial, y Sandra ha contestado correctamente casi todas las cuestiones, obteniendo 21 puntos de un total de 23 posibles respuestas correctas a las preguntas que se formulan sobre cuestiones pragmáticas en este módulo de la prueba.

Las medidas donde se ha visto más impacto son las que hacen referencia a la observación de la conversación de los niños en grupo. Claramente, los niños muestran más habilidades de negociación, de toma de turnos, de propuesta acuerdos, y de resolución de solapamientos después de la intervención, respecto a la evaluación inicial. Concretamente, los niños han obtenido puntuaciones máximas en todas las conductas no verbales (dirigir su mirada a quien habla, tener una actitud corporal adecuada con la escucha y acompañar el discurso del compañero con gestos y expresiones faciales acordes con el discurso). Además, respecto a muchas conductas verbales, también se han podido observar puntuaciones máximas para todos los niños, como la producción de intervenciones correctas desde un punto de vista léxico y semántico, el inicio de turnos, y la conducta de completar el argumento de otro. Sin embargo, algunas de las conductas observadas no han obtenido una buena valoración en la evaluación final, como es el caso de hacer referencia a intervenciones previas de los compañeros, o hacer resúmenes y síntesis. Otras acciones que no están presentes en las conversaciones son el uso por parte de los niños de la lengua vehicular de la escuela, el catalán, siendo el castellano la lengua que utilizan, que es su lengua materna.

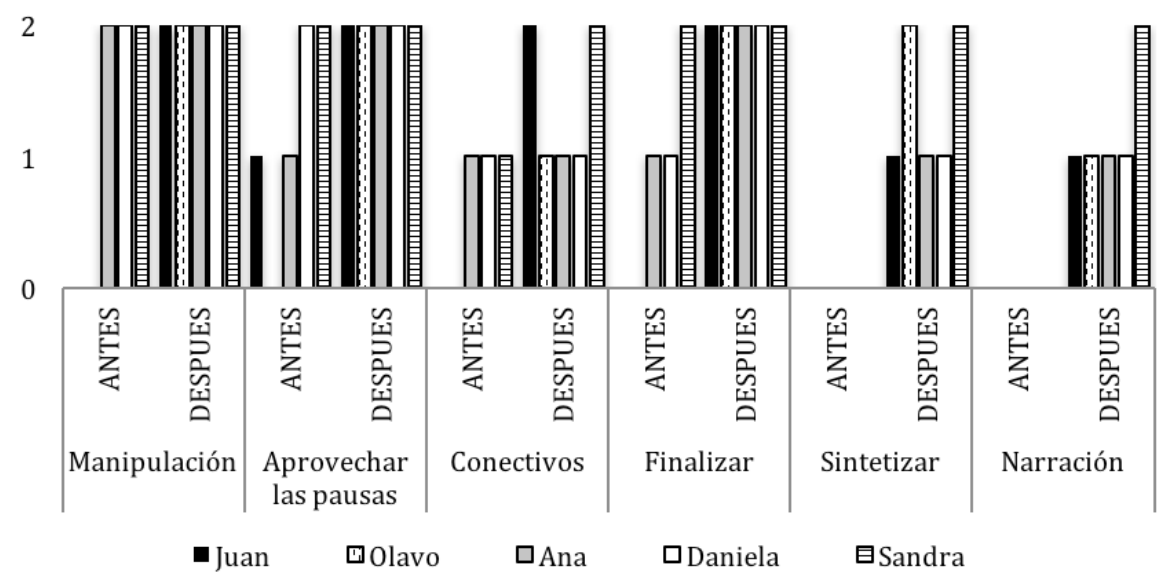

Figura 1. Resultados de las categorías observadas y analizadas durante la actividad de mirar cuentos con la investigadora antes y al final de la intervención. Las categorías han sido analizadas de acuerdo con la frecuencia de emisión: 0: no ha sido observada la categoría; 1 : se puede observar la categoría algunas veces; 2 : se puede observar la categoría sistemáticamente.

En la Figura 1 se presentan las categorías de análisis valoradas durante las actividades de mirar cuentos con la investigadora, antes y al final de la intervención. Las categorías utilizadas fueron: 1. Manipulación; 2. Aprovechar las pausas para hablar; 3. Conectivos; 4. Finalizar; 5. Sintetizar, y 6. Narración. Se puede observar que los 
niños mejoran en casi todas las categorías puntuadas en la observación final respecto a la inicial. Las medidas donde se observa más cambio son las que hacen referencia a la categoría de narración y de hacer síntesis. Respecto a la categoría de manipulación, se puede observar que, antes de la intervención, únicamente tres de los cinco niños (Ana, Daniela y Sandra) manipulaban sistemáticamente los libros, y después de la intervención todos ellos puntúan positivamente en este indicador. En relación con la categoría de hablar durante las pausas en cada página, se observa que inicialmente únicamente dos de los niños hablaron sistemáticamente (Daniela y Sandra), dos de ellos hablaron en algunas pausas (Juan y Ana), y Olavo no habló ninguna vez. Después de la intervención, todos ellos hablaron sistemáticamente durante las pausas. Sobre el uso de conectivos en la lectura, tres niños utilizaron los conectivos algunas veces antes de la intervención (Ana, Daniela y Sandra), después de la intervención Juan y Sandra los utilizaron sistemáticamente, y todos los otros algunas veces. En cuanto a la habilidad de finalizar la lectura, antes de la intervención, Sandra finalizaba sus lecturas sistemáticamente, Ana y Daniela lo hacían algunas veces, y después de la intervención todos ellos finalizaron sus lecturas. Finalmente, respecto a las categorías de narración y de hacer síntesis, se puede observar que antes de la intervención ningún niño demostraba habilidades narrativas o de síntesis, y después de la intervención se observó la presencia de estas habilidades en todos los niños, al menos algunas veces.

\section{Discusión}

El objetivo de la presente investigación era valorar los posibles efectos de una intervención centrada en la familia (Escorcia et al., 2016) para la promoción de avances en las habilidades conversacionales y de lengua oral de niños de cinco años. Además, se han valorado también aspectos importantes de las rutinas de las familias y la manera como los padres y las madres enseñan lengua oral a los niños, teniendo como base el instrumento observacional EVALOF (Domeniconi, Gràcia, Benitez, \& Vessoni, 2017). Un segundo objetivo era detectar aspectos mejorables del propio instrumento para construir un instrumento fiable que sea sensible a detectar al menos una parte importante de la complejidad implicada en la interacción comunicativa entre adultos y niños de una familia.

Un primer resultado que los datos ponen de manifiesto es que las familias en general no aprovechan las rutinas diarias para hablar con sus hijos, reservando los momentos de traslado en general, cuando van de la escuela hacia casa, o de casa a algún otro sitio, para conversar. Únicamente una de las familias, en las que todos comen o cenan juntos, aprovecha estos momentos para hablar con ellos. Sobre el instrumento que hemos utilizado para valorar las interacciones comunicativas, la EVALOF (Domeniconi, Gràcia, Benitez, \& Vessoni, 2017), las cuatro familias inicialmente obtuvieron unas puntuaciones medias que no superaban el 50\% de la máxima puntuación prevista por el instrumento, aunque por razones distintas, como los padres de los hermanos gemelos que tenían un estilo de interacción más directivo, con puntuaciones bajas en los ítems como facilitar que los niños hablen, posibilitar que los niños participen, dejar que los niños gestionen sus turnos. Por otro lado, los padres de las niñas participantes las dejaban hablar sin casi ningún tipo de interacción o ayuda, 
lo que se tradujo en puntuaciones bajas en ítems como enseñar a pedir información, enseñar a hacer síntesis, y pedir autovaloraciones. Así mismo, en todas las familias se han observado avances en la evaluación final, también de manera distinta en cada una de ellas, mejorando especialmente en los ítems con una puntuación inicial muy baja, lo cual indica que han conseguido modificar sus rutinas a partir de las decisiones tomadas conjuntamente en las reuniones sobre la modificación e introducción de estrategias, de manera que les ha permitido incorporar a los niños todavía más en las conversaciones, ya sea dejándolos hablar más, o intentando intercambiar turnos de manera más equilibrada con ellos.

Sobre el uso de la EVALOF para medir los efectos de la intervención sobre las interacciones comunicativas de los padres con sus hijos, el análisis de los datos pone de relieve que la escala puede ser adecuada para medir con detalle muchos aspectos de la manera cómo interactúan padres y madres con la intención de enseñar la lengua oral, y que el instrumento puede ser útil para la planificación de procesos de asesoramiento a familias, como ocurre con la escala original, la EVALOE (Gràcia et al., 2015). Por otra parte, el instrumento EVALOF (Domeniconi, Gràcia, Benitez, \& Vessoni, 2017) necesita una validación para que sea considerado fiable para la valoración de los procesos comunicativos que tienen lugar en el contexto familiar.

Sobre la cuestión de los cambios observados en el repertorio comunicativo de los niños, se pone de relieve que los cinco han mejorado todos los aspectos del repertorio comunicativo, tanto en las pruebas estandarizadas, como en el análisis de la situación conversacional con los pares, y en el análisis de la observación de la situación de mirar cuentos con la investigadora, tal como se recoge en la Tabla 4 y en la Figura 1. Aunque el análisis multimodal sea fiable para la interpretación de los resultados, los resultados no son conclusivos sobre la función de la intervención en el avance del repertorio lingüístico de los niños, puesto que el diseño de la investigación no permite hacer afirmaciones de manera contundente sobre los efectos del desarrollo de los niños gracias a su participación en los contextos habituales, como la escuela, en los resultados observados. Sin duda, investigaciones futuras con un diseño similar al que hemos presentado, $u$ otras con diseños que tengan grupos de comparación, o con mayor número de mediciones durante el proceso de intervención, contribuirán a confirmar estos resultados preliminares.

Los resultados obtenidos, una vez más, reflejan la importancia de la interacción comunicativa entre adultos y niños en contexto familiar, y también el potencial de las actividades de lectura compartida como rutina en contexto familiar para promover avances en el repertorio comunicativo de los niños. Los resultados coinciden con investigaciones anteriores que han valorado sistemáticamente la función de la lectura compartida para la promoción de avances en el vocabulario (Hargrave \& Sénéchal, 2000; Bus, van Ijzendoorn, \& Pellegrini, 1995), la comprensión de los textos, producción de narraciones, y las habilidades comunicativas en general de los niños participantes (Flores, Pires, \& Souza, 2014; Lever \& Sénéchal, 2011).

La mayoría de aspectos incluidos en la intervención llevada a cabo con las familias son coherentes con intervenciones que siguen un modelo enfocado a la familia (Dalmau, Balcells-Balcells, Giné, Cañadas, Casas, Salat, Farré, \& Calaf, 2017), por ejemplo, la escucha activa, la participación activa de los padres y madres, tanto en relación con las pautas $\mathrm{u}$ orientaciones sobre las interacciones, como el uso o no de las pautas propuestas, y el hecho de que toda la intervención era implementada por ellos en sus casas (Espe-Sherwindt, 2008; Espe-Sherwindt \& Serrano, 2016). 
La intervención planteada supone que los adultos que interactúan con los niños son adultos significativos, que conocen a los niños, sus rutinas y sus actividades preferidas, confiriendo naturalidad y funcionalidad a las situaciones. Sin embargo, es necesario destacar que la intervención llevada a cabo no ha explotado todas las características de un enfoque centrado en la familia, especialmente al centrarse en una rutina concreta y en el desarrollo específico de la competencia comunicativa del niño (Dunst, Trivette, \& Hamby, 2007; Espe-Sherwindt, 2008; Division for Early Childhood, 2014), por lo que se ha planteado como una intervención siguiendo un modelo enfocado en la familia, como un paso previo para alcanzar las intervenciones centradas en la familia.

Sin duda, la intervención ha sido breve, planificada para ser llevada a cabo en siete reuniones, además de la evaluación inicial y final. La brevedad de la intervención puede ser interpretada como un elemento positivo en el momento de interpretar los resultados, teniendo en cuenta la necesidad de intervenciones de bajo costo, tanto desde el punto de vista del tiempo invertido por las familias, como por la necesidad de profesionales con formación especializada para ayudar a las familias en aspectos vinculados a la lengua oral. Por otra parte, quizás una intervención más extendida en el tiempo podría producir resultados más significativos. Otro punto que es necesario tener en cuenta es el repertorio inicial de los niños. Aunque cuatro de los cinco niños presentaban niveles de lenguaje por debajo de lo esperado para la edad, de acuerdo con los parámetros de las pruebas estandarizadas, y también muy por debajo en los instrumentos de observación construidos en la investigación, se trata de niños con desarrollo típico, siendo la intervención propuesta considerada como una intervención temprana y preventiva. Entendemos que futuras investigaciones pueden contribuir a la valoración de la eficacia de una intervención de estas características para niños con algún tipo de trastorno comunicativo o en el desarrollo en la línea de trabajos como el de Gràcia (2001), con madres de niños con síndrome de Down.

\section{Bibbliografía}

Benitez, P. \& Domeniconi, C. (2012). Verbalizações de familiares durante aprendizagem de leitura e escrita por deficientes intelectuais. Estudos de Psicologia, 29(4), 553-562.

Benitez, P. \& Domeniconi, C. (2014). Capacitação De Agentes educacionais: proposta de desenvolvimento de estratégias inclusivas. Revista Brasileira de Educação Especial, 20, 371-386.

Bus, A. G., van Ijzendoorn, M. H., \& Pellegrini, A. D. (1995). Joint Book Reading Makes for Success in Learning to Read: A Meta-Analysis on Intergenerational Transmission of Literacy. Review of Educational Research, 65(1), 1-21.

Colmar, S. H. (2014). A parent based book-reading intervention for disadvantaged children with language difficulties. Child Language Teaching and Therapy, 30 (1), 79-90.

Dalmau, M., Balcells-Balcells, A., Giné, C., Cañadas, M., Casas, O., Salat, Y., Farré, V., \& Calaf, N. (2017). Cómo implementar el modelo centrado en la familia en la intervención temprana. Anales de psicología, 33 (3), 641-651.

Dempsey, I., \& Keen, D. (2008). A review of processes and outcomes in family centered services for children with a disability. Topics in Early childhood Special Education, 28(1), 42-52. http://doi.org/10.1177/0271121408316699 . 
Dickinson, D. K., \& Tabors, P. O. (2001). Beginning language with literacy: Young children learning at home and school. Baltimore, MD: Brookes Publishing.

Division for Early Childhood. (2014). DEC recommended practices in early intervention/ early childhood special education 2014. Retrieved from http://www.dec-sped.org/recommendedpractices

Domeniconi, C., Gràcia, M., Benitez, P., \& Vessoni, J. (2017). Adaptação da Escala de Avaliação do Ensino de Linguagem Oral Em Contexto Escolar (EVALOE) Para Seu Uso Em Contexto Familiar (EVALOF). Revista de Psicologia da Criança e do Adolescente, 8 (1), 65-80.

Dunst, C.J., Trivette, C. M., \& Hamby, D. W. (2007). Meta-analysis of familycentered helpgiving practices research. Developmental Disabilities Reseach Reviews, 13, 370-378. http://doi.org/10.1002/mrdd.20176

Escorcia Mora, C. T., García Sánchez, F. A., Orcajada Sánchez, N., \& Sánchez López, M. C. (2016). Perspectiva de las prácticas de atención temprana centradas en la familia desde la logopèdia. Revista de Logopedia, Foniatría y Audiología, 36 (4), 170-177.

Espe-Sherwindt, M. (2008). Family-centered practice: A collaborative, competency enhancing, evidence-based model. Support for Learning, 23 (3), 137-143

Espe-Sherwindt, M. \& Serrano, A. M. (2016). It takes two: The role of famiy-centered practices. Revista de Logopedia, Foniatría y Audiologia, 36, 162-169.

Flores, E. P., Pires, L. F., \& Souza, C. B. A. D. (2014). Dialogic reading of a novel for children: Effects on text comprehension. Paidéia, 24(58), 243-252. doi: 10.1590/198243272458201412

García-Sánchez, F. AS., Escorcia Mora, C. T., Sánchez López, M. C., Orcajada Sánchez, Noelia, \& Hernández-Pérez, E., (2014). Atención temprana centrada en la familia. Siglo Cero, 251, 6-27.

Gortmaker, V. J., Daly, E. J., McCurdy, M., Persampieri, M. J., \& Hergenrader, M. (2007). Improving reading outcomes for children with learning disabilities: using brief experimental analysis to develop parent tutoring interventions. Journal of Applied Behavior Analysis, 40, 203-221.

Gràcia, M. (2001). Intervención naturalista en la comunicación y el lenguaje en ámbito familiar: un estudio de cuatro casos. Infancia y Aprendizage, 24, 307-324.

Gràcia, M., Galván-Bovaira, M. J., Sánchez-Cano, M., Vega, F., Vilaseca, R., \& Rivero, M. (2015). Evaluación de la enseñanza de la lengua oral. Escala EVALOE. Barcelona: Graó.

Gràcia, M., Vega, F., \& Galván-Bovaira, M. J. (2015). Developing and testing EVALOE: a tool for assessing spoken language teaching and learning in the classroom. Child Language Teaching Therapy, 31 (3), 287-304.

Gurgueira, L. H., \& Cortegoso, A. L. (2008). Avaliação de um programa de ensino para capacitar mães como agentes favorecedoras do estudar. Psicologia da Educação, 27(1), 5-30.

Hancock, T.B., \& Kaiser, A.P. (2002). The effects of trainer-implemented enhanced milieu teaching on the social communication of children who have autism. Topics in Early Childhood Special Education, 22(1), 39-54.

Hargrave, A. C. \& Sénéchal, M. (2000). A book story reading intervention with preschool children who have limited vocabularies: The benefits of regular reading and dialogic reading. Early Childhood Research Quarterly, 15(1), 75-90. doi: 10.1016/S08852006(99)00038-1.

Hart, B., \& Rogers-Warren, A. (1978). A milieu approach to teaching language. In R. Schiefelbusch (Ed.), Language intervention strategies (pp. 193-235). Baltimore, MD: University Park Press. 
Hart, B. M., \& Risley, T. R. (1995). Meaningful differences in the everyday experiences of young American children. Baltimore, MD: Paul . Brookes.

Hoff, E. (2012). Language Development. Belmont, CA. Wadworth Publishing Co Inc.

Koegel, R.L., O’Dell, M.C., \& Koegel, L.K. (1987). A natural language teaching paradigm for nonverbal autistic children. Journal of Autism and Developmental Disorders, 17(2), 187-200.

Lever, R., \& Sénéchal, M. (2011). Discussing stories: On how a dialogic reading intervention improves kindergartners' oral narrative construction. Journal of Experimental Child Psychology, 108, 1-24. doi: 10.1016/j.jecp.2010.07.002.

McWilliam, R. (2016). The Routines-Based Model for supporting speech and language. Revista de Logopedia, Foniatría y Audiología, 36 (4), 178-184.

Milburn, T.F., Girolametto, L., Weitzman, E., \& Greenberg, J. (2014). Enhancing preschool educator's ability to facilitate conversations during shared book reading. Journal of Early Childhood Literacy, 14 (1), 105-140.

Stocco, C.S., \& Thompson, R. H. (2015). Contingency analysis of caregiver behavior: implications for parent training and future directions. Journal of Applied Behavior Analysis, $48,1-19$.

Tomasello, M. (2003). Constructing a language: A usage-based theory of language acquisition. Cambridge: Harvard University Press.

Vilaseca, R. \& del Rio, M. J. (2004). Language acquisition by children with Down syndrome: A naturalistic approach to assisting language acquisition. Child Language Teaching \& Therapy, 20, 163-180

Yin, R. K. (2008). Case Study Research: Design and Methods. London: SAGE.

\section{Anexo}

Detalle de cada una de las reuniones de seguimiento (la señal + indica los retos que los padres han relatado como positivos y alcanzados por ellos conjuntamente con sus hijos):

\begin{tabular}{ll} 
Ana & \multicolumn{1}{c}{ Daniela Juan y Olavo Sandra } \\
$\begin{array}{l}\text { 1.Contacto } \\
\text { Grupo }\end{array}$ & $\begin{array}{l}\text { Objetivos: presentación, firmar documentos, concretar fechas, aclarar } \\
\text { dudas. }\end{array}$ \\
$\begin{array}{l}\text { 2.Entrevista } \\
\text { sobre rutinas } \\
\text { Individual }\end{array}$ & $\begin{array}{l}\text { Objetivos: recoger informaciones sobre las rutinas de las familias, con } \\
\text { el objetivo de conocer las actividades de comunicación que la madre y } \\
\text { el padre llevan a cabo con sus hijos. }\end{array}$ \\
$\begin{array}{l}\text { 3. Reunión de } \\
\text { asesoramien- } \\
\text { to_1 }\end{array}$ & $\begin{array}{l}\text { Objetivos: empezar las reflexiones sobre como hablan entre ellos en } \\
\text { casa y especialmente como miran cuentos juntos. Se utilizan vídeos o }\end{array}$ \\
$\begin{array}{l}\text { Individual } \\
\text { 4. Reunión de } \\
\text { asesoramien- } \\
\text { to_2 }\end{array}$ & $\begin{array}{l}\text { Objetivos: presentar y discutir un conjunto de siete sugerencias /es- } \\
\text { Individual }\end{array}$ \\
$\begin{array}{l}\text { trategias, teniendo en cuenta las rutinas de cada familia, para el mo- } \\
\text { mento de mirar cuentos con los niños, y también un calendario donde }\end{array}$ \\
$\begin{array}{l}\text { se podrían anotar los días en que se realiza la actividad de mirar cuen- } \\
\text { tos. }\end{array}$
\end{tabular}


5. Reunión de asesoramiento_3

Individual
Objetivos (los mismos hasta reunión 5): discutir el uso de las sugerencias. Puntos positivos $(+)$ y dificultades principales presentadas por cada familia o próximos retos $(\mathrm{PR})$

$\begin{array}{llll}+ \text { seguir el } & \text { + están leyendo } & \text { + han empezado } & \text { + están ley- } \\ \text { interés de la } & \text { más } & \text { a leer } & \text { endo más } \\ \text { niña } & \text { Próximo reto: inter- } & \text { Próximo reto: } & \text { Próximo } \\ \text { Próximo } & \text { cambio de turnos } & \text { conseguir el } & \text { reto: diver- } \\ \text { reto: sacar } & & \text { interés de uno } & \text { sificar los } \\ \text { conclu- } & & \text { de los niños } & \text { temas, ele- } \\ \text { siones y } & & & \text { gir cuentos } \\ \text { síntesis } & & & \text { nuevos }\end{array}$

\begin{tabular}{|c|c|c|c|c|}
\hline $\begin{array}{l}\text { 6. Reunión de } \\
\text { asesoramien- } \\
\text { to } 4 \\
\text { Individual }\end{array}$ & $\begin{array}{l}+ \text { preguntas } \\
\text { abiertas } \\
\text { Próximo } \\
\text { Reto: ex- } \\
\text { pansiones y } \\
\text { cambiar los } \\
\text { temas de los } \\
\text { cuentos }\end{array}$ & $\begin{array}{l}\text { + están leyendo } \\
\text { más } \\
\text { Próximo Reto: in- } \\
\text { tercambio de turnos }\end{array}$ & $\begin{array}{l}\text { + clarificación y } \\
\text { expansiones } \\
\text { Próximo Reto: } \\
\text { leer de manera } \\
\text { más tranquila, } \\
\text { divertida, y un } \\
\text { rato corto }\end{array}$ & Cita médica \\
\hline $\begin{array}{l}\text { 6. Reunión de } \\
\text { asesoramien- } \\
\text { to } 5 \\
\text { Individual }\end{array}$ & $\begin{array}{l}\text { + la niña ha } \\
\text { sacado con- } \\
\text { clusiones } \\
\text { Próximo } \\
\text { Reto: } \\
\text { mantener } \\
\text { y mejorar } \\
\text { vocabulario }\end{array}$ & Cita médica & $\begin{array}{l}\text { + están leyendo } \\
\text { más, los niños } \\
\text { tienen interés, y } \\
\text { es divertido } \\
\text { Próximo Reto: } \\
\text { eliminar frases } \\
\text { más complejas }\end{array}$ & $\begin{array}{l}\text { Celebración } \\
\text { familiar }\end{array}$ \\
\hline
\end{tabular}

7. Reunión final Objetivo: entregar documento de valoración general sobre cada niño; Grupo encias y opiniones sobre el trabajo 